\title{
Proveedores comerciales de revistas electrónicas de ciencias sociales
}

\author{
Por Juan Carlos Martín González y José Antonio Merlo Vega
}

Resumen: Las revistas electrónicas son un recurso excepcional para la difusión de la investigación científica. Se presentan sus principales proveedores comerciales en ciencias sociales, comentando los aspectos más importantes que identifican a cada compañía, como son la trayectoria histórica, los servicios de acceso a las revistas y artículos que ofrece, la cantidad de publicaciones periódicas electrónicas a las que da acceso y las disciplinas en las que están especializados.

Palabras clave: Revistas electrónicas, Proveedores, Ciencias sociales, Editoriales.

\section{Title: Commercial suppliers of electronic journals in the social sciences}

Abstract: Electronic journals are exceptional resources for the dissemination of scientific research. Covered in this paper are the principal commercial suppliers of social science e-journals, with details about each company such as its background, additional services offered, number of journals included in their electronic catalogues and their subject specialisation.

Keywords: Electronic journals, Suppliers, Social sciences, Publishers.

\section{LAS REVISTAS ELEC-} TRÓNICAS se están convirtiendo en un vehículo de difusión de la investigación científica de evidente utilidad. Nadie duda de sus ventajas, que pueden resumirse en: facilidad de acceso, nulos problemas de almacenamiento, mayor difusión, abaratamiento de los costes, diversidad de formatos, ampliación de las posibilidades de consulta, acceso a los documentos de forma independiente, interoperatividad con otros recursos informativos (catálogos, bases de datos), servicios de valor añadido (alertas, dsi, etc.), simplificación de los procesos técnicos y fomento de la cooperación y el intercambio científico.
Existen distintos tipos de proveedores cuya actividad comercial consiste en facilitar el acceso a sus contenidos. En muchos casos se trata de los mismos editores, quienes emplean la edición electrónica de forma complementaria a la impresa. En muchas otras ocasiones los sistemas para acceder a una publicación periódica, o a un artículo de la misma, pasan por los servicios de empresas que actúan como intermediarias, ya sea albergando las revistas en sus servidores o simplemente facilitando el acceso a los mismos. No se pretende en este trabajo realizar una sistematización de las diferentes posibilidades de acceso a una revista electrónica, ya que la idea fundamental es agrupar a sus proveedores comer- ciales en el ámbito de las ciencias sociales, comentando los detalles más importantes de su trayectoria histórica, sus servicios y sus publicaciones.

Por tanto, quedan fueran de este análisis los sistemas públicos de acceso a revistas electrónicas, cada vez más frecuentes, y las compañías que editan o distribuyen de forma comercial publicaciones periódicas en formato electrónico cuya temática se centra en las ciencias puras, las tecnologías o las humanidades. Se ofrece una lista alfabética de editores y distribuidores especializados en ciencias sociales $\mathrm{y}$, además, de empresas que las comercializan, de cualquier disciplina, pero con un porcentaje impor- 
tante de sus catálogos dedicado a las ciencias sociales.

Entre las compañías seleccionadas también se ha decidido incluir los servicios de publicaciones electrónicas ofrecidos por agencias de suscripciones (Ebsco, SwetsBlackwell), agregadores (Ovid) y un socio tecnológico (Ingenta Select), a pesar de tratarse de servicios de intermediación o enlazado, puesto que estos tipos de plataformas se consideran puntos de acceso de uso bastante común y generalizado. Como agregadores se conocen los servicios que presentan determinadas empresas que llegan a acuerdos con los editores para albergar sus revistas y darles acceso desde una interfaz común. Obviamente esos servicios no son editores comerciales propiamente dichos, pero pueden ser considerados proveedores de acceso a publicaciones en línea de ciencias sociales, así como fuentes de información susceptibles de ser consultadas. De este modo se pretende ofrecer una visión general de las diferentes vías de acceso a la información digital en este campo.

Para delimitar las disciplinas que se encuadran dentro de las ciencias sociales se ha partido de las grandes clasificaciones enciclopédicas, como Dewey, CDU o la clasificación de la Biblioteca del Congreso. También se han consultado las distintas divisiones temáticas que los proveedores han realizado para dar acceso a las revistas que comercializan. De igual forma se han empleado repertorios de obras de referencia de ciencias sociales, directorios de internet de estas disciplinas y otras fuentes adicionales. En definitiva, las compañías que a continuación se comentan son aquellas que editan o distribuyen revistas electrónicas de estadística y demografía, ciencias políticas, economía y empresas, derecho, administración pública, trabajo social, pedagogía, psicología, sociología, antropología, comunicación y documentación.

La relación de editoriales se presenta ordenada alfabéticamente, destacando algunas de las direcciones web en las que se puede encontrar información sobre los servicios de acceso a las revistas electrónicas de cada una. Los comentarios que se han redactado para los diferentes proveedores intentan reflejar las cuestiones de mayor interés para los lectores: presencia en el mercado, colecciones, servicios, ámbitos temáticos, cobertura temporal de las colecciones en línea, etc. Es habitual que las empresas se absorban, se fusionen o que cambien de nombre. En este caso se ha optado por remitir a la nueva marca o a la compañía que ha comprado a otra.

\section{-Academic Press}

Véase Elsevier.

\section{—Blackwell Publishing}

Empresa británica fundada en 1939. La actual es fruto de la fusión en 2001 de Blackwell Science y Blackwell Publishers. Como editora está especializada en humanidades, ciencias sociales, economía y tecnologías de la información, aunque en sus catálogos no dejan de lado otras disciplinas. Da acceso en línea a cerca de 650 revistas, de las que un elevado número corresponde a materias englobadas en las ciencias sociales. Ofrece dos modalidades de acceso: una gratuita, para los suscriptores a la versión impresa (aunque sólo para el año en curso y el anterior) y otra no-gratuita con acceso a los archivos a partir de 1997-1998 y, entre otras ventajas, acceso a los preprints de los números en curso. http://www.blackwellpublishing.com

\section{—Brill Academic Publisher}

Compañía holandesa dedicada a la edición científica desde finales del siglo XIX. Edita y distribuye un centenar de revistas en línea, además de monografías y enciclopedias. Sus campos de especialización son la historia, la religión, los estudios islámicos, asiáticos y clásicos. Edita también monografías y revistas sobre otras materias sociohumanísticas. Permite un acceso individual o institucional a sus publicaciones periódicas en línea de modo gratuito a los suscriptores de la versión impresa (de momento sólo a los años anteriores durante el periodo suscrito a la revista en papel). Para la gestión de sus publicaciones electrónicas emplea los servicios de Ingenta Select, quien actúa como socio tecnológico.

http://www.brill.nl

\section{-Cambridge University Press}

A pesar de ser una editorial universitaria ha conseguido un gran prestigio internacional dentro de la edición científica. Su servicio de acceso a las publicaciones periódicas que edita, denominado Cambridge Journal Online, permite consultar el texto completo de cerca de un centenar de revistas de ciencias puras, ciencias sociales y humanidades. El acceso es gratuito para los suscriptores de la revista impresa, y existe la posibilidad de utilizar sus archivos desde el año 1997. Además posee distintas formas para acceder al contenido de las publicaciones que edita. Una de ellas es el índice temático, que es de gran utilidad para conocer y consultar los títulos de cada tema. Permite obtener artículos sueltos mediante el pago de tarifas por visualización por un tiempo limitado.

http://journals.cambridge.org/

\section{—Carfax Publishing}

Véase Taylor \& Francis
Group.

\section{- Catchword}

Véase Ingenta Select. 


\section{Inc.}

\section{—Ebsco Information Services}

Con más de 60 años en el sector de la producción y distribución de publicaciones científicas y bases de datos, Ebsco es hoy una de las empresas líderes del sector. Desarrolla sus servicios de agencia de suscripciones actuando como pasarela entre los usuarios y los editores. Su oferta sobrepasa las 4.500 revistas de todos los campos de la ciencia. El acceso a las publicaciones periódicas lo realiza a través de Electronic Journals Services (EJS), interfaz de consulta que hace posible la interrogación por artículos o por revistas y que, además, permite la tecnología $s f x$.

http://www.ebsco.com

\section{-Elsevier-Science Direct}

Es el servicio de acceso a las publicaciones periódicas en formato electrónico del grupo editorial Elsevier. Su política inicial fue suministrar sólo revistas propias, aunque en los últimos años también ofrece dentro de sus servicios algunas publicaciones de otras editoriales. Su catálogo contiene cerca de 1.700 revistas, de temática multidisciplinar, de las cuales unas 225 están relacionadas con las ciencias sociales. La licencia de Science Direct permite el acceso a artículos publicados a partir de 1998. El formato de sus archivos es siempre pdf. En el año 2002 adquirió las editoriales Academic Press y Harcourt e incorporó sus servicios de publicaciones electrónicas, concretamente Ideal Library, en Science Direct.

http://www.sciencedirect.com

\section{- Emerald}

$M C B$ University Press toma el nombre de Emerald cuando comienza a orientar su negocio hacia las publicaciones electrónicas. Se trata de una de las editoriales con más prestigio en ciencias económicas y empresariales, aunque entre su más de un centenar de revistas también se encuentran algunas de ingeniería o de documentación. Entre sus servicios destaca Emerald Full Text, desde el que se puede consultar el texto íntegro de sus publicaciones, ya sea en html o en pdf abarcando hasta el año 1993. http://www.emerald-library.com

\section{-Frank Cass}

Inicia su trayectoria profesional en 1957 y desde entonces es una de las editoriales comerciales británicas más afamadas de las que se dedican a las publicaciones periódicas científicas de ciencias sociales. Dispone de más de sesenta títulos en línea. En la actualidad permite el acceso gratuito a los suscriptores de las versiones impresas, durante el periodo suscrito, empleando un sistema de identificación mediante nombre de usuario y contraseña.

http://www.frankcass.com/jnls/

\section{-Gordon and Breach}

\section{Véase Taylor \& Francis Group.}

\section{- Harcourt}

\section{Véase Elsevier.}

\section{-Ingenta Select}

Este es un buen ejemplo de la mutabilidad de las empresas dedicadas a la información electrónica. Adquiere en 2002 Catchword, una de las principales empresas de acceso a publicaciones científicas. En enero de 2003 toma el nombre actual, aunque mantiene los mismos servicios. Actúa como socio tecnológico, es decir, que ofrece su tecnología a distintas editoriales que deseen poner en línea sus publicaciones. Los editores que trabajan con este servicio están especializados en las distintas disciplinas del conocimiento con cerca de 5.000 revistas albergadas en los servidores de Ingenta Select. Su mayor crecimiento se produjo sobre todo a partir de la compra de Catchword, ya que el catálogo se amplió considerablemente. Ade- más de pdf, muchas de las revistas a las que se accede desde este servicio también emplean el formato Real page.

http://www.ingentaselect.com

\section{—John Wiley \& Sons Ltd.}

Éste es uno de los ejemplos más claros de la adaptación de los editores convencionales al mercado de la información electrónica, ya que se trata de una empresa creada a principios del siglo XIX que, a lo largo del XX, se especializó en publicaciones científicas. En la actualidad a través de su servicio $I n$ terscience da acceso a más de 300 revistas de temática multidisciplinar, de las que un centenar es de ciencias sociales: economía, educación, derecho y psicología fundamentalmente. Ofrece diferentes variantes de licencias, de las cuales depende la cobertura para el acceso a años anteriores.

http://www.interscience.wiley.com

\section{-Kluwer}

Empresa holandesa, creada a finales del siglo XIX y que en estos momentos está plenamente consolidada, contando con una amplia presencia en el sector. Se trata de un editor de monografías, obras de referencia y revistas científicas, aunque no está especializado en una materia concreta, sino que edita títulos multidisciplinares. En la actualidad su servicio Kluwer On Line permite el acceso a cerca de 800 publicaciones periódicas electrónicas, de las que unas 300 tratan sobre materias de ciencias sociales y están en línea. Actualmente propone dos modelos de adquisición de sus revistas electrónicas: acceso gratuito a los suscriptores de sus ediciones impresas, solamente para los artículos publicados durante el año en curso y con acceso restringido a un número muy limitado de direcciones ip y, por otra parte, existe la posibilidad de un acceso no gratuito, que da derecho a la consulta de los artículos publica- 
dos durante el periodo suscrito a la versión impresa de la revista.

http://www.kluweronline.com

\section{-Lawrence Erlbaum Asso- ciates $($ LEA)}

Editor estadounidense de libros, software y revistas científicas. Dispone de una colección de cerca de 85 publicaciones periódicas, especializadas en varias ramas del saber, entre las que se encuentran algunas de las ciencias sociales. Su servicio de acceso en línea a las revistas científicas que edita se realiza a través de la empresa antiguamente denominada Catchword, hoy conocida como Ingenta Select. Esta opción es la misma que siguen muchos pequeños editores, para quienes es más rentable disponer de un socio tecnológico para dar acceso a sus publicaciones. Su política de suscripciones ha variado respecto a años anteriores y ya no concede la consulta gratuita de la versión electrónica para quienes ya reciban la edición impresa. Otro de sus servicios es el envío de los sumarios por correo electrónico.

http://www.erlbaum.com

\section{lliams}

-Lippincott Wilkins and Wi-

Véase Ovid.

—MCB University Press

Véase Emerald.

\section{-Ovid Technologies}

El negocio tradicional de Ovid siempre ha sido la distribución de bases de datos, ámbito en el que ha conseguido un papel muy importante, sobre todo a partir de la compra de SilverPlatter, pero también destaca como agregador, dando acceso a cerca de 800 revistas científicas electrónicas de temática multidisciplinar, sobresaliendo en el ámbito de la medicina. No obstante, en su servicioJournals@Ovid puede encontrarse un elevado número de publicaciones de ciencias sociales. En el año 2003 obtiene la exclusividad de los títulos de Lippincott Wilkins and Williams. Las tecnologías desarrolladas por Ovid permiten la integración de los sistemas de acceso a las revistas electrónicas y sus bases de datos bibliográficas.

http://www.ovid.com

\section{—oxford University Press}

Otra de las editoriales universitarias de mayor arraigo internacional (tiene distintas sedes en el Reino Unido, EUA y Japón) es ésta que, como no podía ser de otra manera, también tiene un servicio de acceso en línea a sus publicaciones periódicas, denominado Oxford Journals desde el que se pueden consultar los textos completos de casi dos centenares de revistas científicas, muchas de ellas de carácter tecnológico, pero con otra elevada cantidad de títulos de disciplinas humanísticas y de ciencias sociales. El formato elegido para los artículos de las revistas es pdf. En la actualidad OUP ofrece acceso en línea gratuito a los suscriptores de su publicación y la cobertura temporal de acceso a archivos de años anteriores varía según las características específicas de cada publicación.

http://www.oup.co.uk

\section{—Project Muse}

Proyecto de la Universidad Johns Hopkins iniciado en 1995 y a través del cual se pueden consultar cerca de 200 revistas académicas de calidad, publicadas por una treintena de editores de distinta procedencia. Es posible suscribirse por áreas temáticas, la de ciencias sociales contiene unos 120 títulos, todos en lengua inglesa. Incluso se permite la suscripción a un único título, aunque en este caso se limita el tipo de acceso y se establecen tarifas diferentes.

http://muse.jhu.edu

\section{—Psychology Press}

\section{Véase Taylor \& Francis Group.}

\section{—Routledge}

Véase Taylor \& Francis
Group.

\section{—Sage Publications}

Con una presencia en el mercado desde hace más de 40 años, esta compañía destaca en la producción y distribución de revistas especializadas en las distintas ramas de las ciencias sociales. Sobre todo despunta en educación, y es además editora de un elevado número de monografías sobre temas relacionados con esta materia. En la actualidad dispone de un fondo editorial de más de 350 revistas y ofrece las versiones en línea de sus publicaciones de forma gratuita, junto con la suscripción a la edición impresa. También permite el acceso a volúmenes editados con anterioridad, aunque sólo del periodo suscrito.

http://www.sagepub.com

\section{—Springer Verlag}

Éste es otro de los ejemplos más ilustrativos de la evolución del mercado de las publicaciones electrónicas, ya que su origen se encuentra en una librería abierta en Berlín en 1842. En estos momentos actúa como editor comercial que distribuye en línea sus propias publicaciones, cerca de 500, a través de su servicio Springer Link. Los artículos de sus revistas pueden ser consultados en html, pdf o post script, incluyendo los publicados desde 1996. También posee un servicio de preprints denominado On Line First Publications. Otra particularidad de esta compañía es que da especial importancia a los $d o i$, permitiendo realizar búsquedas a través de este código de identificación en sus artículos.

http://link.springer.de 


\section{—Swets Blackwell}

Swets es una empresa pionera como agencia de suscripciones a publicaciones periódicas. Lleva más de cien años en el mercado, con sucesivas alianzas o adquisiciones de otras compañías, como ocurrió en el año 2000, cuando compró la parte del negocio de suscripciones de Blackwell Information Services, tomando la denominación actual de Swets Blackwell $B$. $V$. Como compañía de acceso a revistas electrónicas actúa haciendo de pasarela, permitiendo en estos momentos consultar más de 7.000 revistas de unos 230 editores, a través de su servicio SwetsWise. Dado el volumen de publicaciones que manejan, el contenido de las mismas es variado, y se pueden encontrar ejemplos de publicaciones científicas de cualquier disciplina. Sus servicios permiten el enlazado con bases de datos, el uso de la tecnología $s f x$ y el empleo de doi.

http://www.swetswise.com
Editorial multidisciplinar británica con más de 200 años de presencia en el mercado y que presta un especial interés a las ciencias sociales. En su amplio catálogo se encuentran cerca de 900 publicaciones periódicas, de las cuales la práctica totalidad está disponibles en línea. Su crecimiento en los últimos años se debe, entre otros factores, a la fusión o adquisición de editoriales de tradición como Gordon \& Breach, Carfax Publishing, Psychology Press o Routledge. En la actualidad, el grupo Taylor \& Francis está formado por doce editoriales distintas, tanto especializadas en revistas como en obras de referencia o monografías. Para dar acceso a las versiones electrónicas de sus publicaciones emplean los servicios de Metapress como socio tecnológico y ofrecen acceso en línea gratuitamente junto con la suscripción impresa así como acceso a archivos de años anteriores durante el periodo suscrito. http://www.tandf.co.uk
Fernández Sánchez, Elena; Fernández Morales, Isabel. "Consideraciones sobre la edición electrónica de revistas en internet”. En: El profesional de la información, 2000, marzo, v. 9 , n. 3, p. 4-12.

Huber, Charles F. "Electronic journal publishers: a reference librarian's guide". En: Issues in science and technology librarianship, 2000, summer. Consultado en: 05-06-03.

http://www.library.ucsb.edu/istl/00summer/article2.html

Marcos Mora, Mari Carmen. "La revista electrónica y su aceptación en la comunidad científica”. En: El profesional de la información, 2000, mayo, v. 9, n. 5, p. 4-14.

Martín González, Juan Carlos; Merlo Vega, José Antonio. "Las revistas electrónicas: características, fuentes de información y medios de acceso". En: Anales de documentación, 2003, n. 6, p. $155-186$

Juan Carlos Martín González, Swets-Blackwell.

jmartin@es.swetsblackwell.com

José Antonio Merlo Vega, Universidad de Salamanca. merlo@usal.es 